\title{
Gender difference in plasminogen activator inhibitor-1 activity in patients with type 2 diabetes with and without albuminuria, a matched case-control study
}

\author{
Mehrnaz Imani, Soghra Rabizadeh, Manouchehr Nakhjavani, Payam Hashemi, \\ Shaghayegh Pezashki, Reyhane Hizomi, Alireza Esteghamati, Hossein Mirmiranpour* \\ ${ }^{1}$ Endocrinology and Metabolism Research Center (EMRC), Vali-Asr Hospital, Tehran University \\ of Medical Sciences, Tehran, Iran
}

Corresponding author: Hossein Mirmiranpour, Endocrinology and Metabolism Research Center (EMRC), Vali-Asr Hospital, Tehran University of Medical Sciences, Tehran, Iran

Submission Date: January $19^{\text {th }}$, 2019. Acceptance Date: July $28^{\text {th }}$, 2019. Publication Date: July $31^{\text {st }}, 2019$.

Citation: Imani M., Rabizadeh S., Nakhjavani M., Hashemi P., Pezashki S., Hizomi R., Esteghamati A., et al.: Gender difference in plasminogen activator inhibitor-1 activity in patients with type 2 diabetes with and without albuminuria, a matched case-control study. Functional Foods in Health and Disease 2019; 9(7): 484-493. DOI: https://doi.org/10.31989/ffhd.v9i7.595

\begin{abstract}
Background: Women with type 2 diabetes are more susceptible to coagulopathy disorders and endothelial dysfunction. One possible explanation is the effects of different sex hormones in inflammatory conditions. Increased plasminogen activator inhibitor-1 (PAI-1) activity has been observed as a possible predisposing factor for coagulopathy disorders and endothelial dysfunction. However, the effect of gender on PAI-1 in patients with type 2 diabetes (T2D) and albuminuria has not been studied sufficiently.
\end{abstract}

Objectives: In this study, we examined whether changes of PAI-1 activity according to the albuminuria state in patients with type 2 diabetes are different in males and females.

Materials and Methods: A matched case-control study was performed among participants with T2D, as 38 microalbuminuric patients were matched with 38 normoalbuminuric patients who were similar in age and body mass index (BMI). PAI-1 activity was compared between the two groups with and without gender stratification.

Results: PAI-1 activity in microalbuminuric women was higher in comparison to that of the normoalbuminuric controls (P-value < 0.05). There was no significant difference in PAI-1 activity between macroalbuminuric and normoalbuminuric men. In women with type 2 diabetes and 
albuminuria, PAI-1 activity was independently and significantly associated with urinary albumin excretion.

Conclusions: Gender differences in PAI-1 activity, seen in the early stages of diabetic nephropathy, are a possible explanation for the higher incidence of vasculopathy in women with type 2 diabetes

Keywords: plasminogen activator inhibitor-1; coagulopathy; microalbuminuria; type 2 diabetes; gender

\section{INTRODUCTION}

PAI-1 is an inhibitor of fibrinolysis that may predict future risk of thrombosis and myocardial infarction [1, 2]. Elevated PAI-1 levels have been associated with several metabolic cardiovascular risk factors such as obesity, insulin resistance, and increased levels of glucose, insulin, proinsulin, and blood lipids $[3,4]$. The exact nature of these relationships has not been established, and the importance of each factor may differ with gender [5, 6]. Increased PAI-1 levels are caused by PAI1 hyper-production within the vascular compartment. Endothelium is sensitive to thrombin, angiotensin IV, cytokines, biologically active lipids and oxidative stress effect, whereas on the contrary insulin inhibits cytokine-induced PAI-1 production [7]. With that being said, PAI-1 deficiencies appear to be quite rare. Affected individuals exhibit mild to moderate bleeding symptoms, including epistaxis, menorrhagia and delayed bleeding after trauma or surgical procedures [8]. Moreover, PAI-1 contributes to diabetic nephropathy by regulating TGF- $\beta$ and renal extracellular matrix production, which may be a therapeutic target in diabetic nephropathy [9].

Elevated PAI-1 activity is associated with albuminuria in type 2 diabetes, suggesting that PAI1 activity may correlate with increased vascular injury [10]. In addition, gender specific association between clotting parameter and cardio metabolic risk factors in patients with type 2 diabetes has been previously examined [11].

To our knowledge, gender specific variation in PAI-1 activity and albuminuria has not been established. The purpose of the present study was to determine the effect of gender on PAI-1 activity in patients with type 2 diabetes with established microalbuminuria in comparison to normoalbuminuric patients.

\section{MATERIALS AND METHODS:}

This was a case-control study conducted between January 2015 and July 2015. The study population consisted of 76 participants, including 38 macroalbuminuric and 38 normoalbuminuric patients with type 2 diabetes. Cases and controls were matched by sex ( 22 males and 16 females in case group and 22 males and 16 females in control group), as well as by age and BMI. The diabetic patients were recruited from the diabetes clinic of Vali-Asr hospital affiliated with Tehran University of Medical Sciences. Diabetes was diagnosed according to guidelines set by American Diabetes Association [12]. The ADA criteria were also used for cutoff values of 24-hour urine collection to diagnose microalbuminuria as 30-299 $\mathrm{mg} / 24 \mathrm{~h}$ albumin excretion and normoalbuminuria as $<30 \mathrm{mg} / 24 \mathrm{~h}$ albumin excretion [13]. In choosing the case group, established microalbuminuria during the 24-hour urine collection was taken into consideration. Exclusion 
criteria for type 2 diabetes patients included dialysis, glomerulonephritis, AST>30 U/L and/or ALT> $40 \mathrm{U} / \mathrm{L}$, hematologic disease, congestive heart failure, stroke, autoimmune disease, hormone replacement therapy, oral contraceptive pill use, pregnancy, hospital admission in the previous six months, onset age of diabetes less than 30 or more than 70 years, diabetic ketoacidosis and nonketotic hyperosmolar diabetes. Demographic and anthropometric data, including age, sex, height, weight and duration of diabetes, were recorded. Body mass index $\left(\mathrm{Kg} / \mathrm{m}^{2}\right)$ was calculated using the Quetelet formula. Blood pressure was measured in the sitting position and remeasured twice after 5 minutes on average. A thorough investigation of diabetic complications was carried out in patients with diabetes. Ischemic heart disease (IHD) was defined as previously known coronary artery disease, positive exercise stress test or at-rest electrocardiographic finding suggestive for IHD. A complete drug history of patients was obtained for hypoglycemic drugs, insulin therapy, antihypertensive drugs, anticoagulant and lipid-lowering drugs. Hypoglycemic drugs consisted of glyburide, metformin and repaglinide. Antihypertensive drugs included captopril, enalapril, losartan, atenolol, diltiazem and amiloride. All participants were treated with aspirin. Lipid-lowering drugs were atorvastatin and gemfibrozil. No patient was treated with niacin, warfarin or heparin. All participants gave written informed consent before participation in the study. This study complied with the principle of the declaration of Helsinki. In addition, the local ethics review committee of Tehran University of Medical Sciences approved the study protocol.

Fasting blood samples were taken after 12 hours of overnight fasting. Fresh blood was used for the measurement of fasting blood sugar (FBS), hemoglobin A1C (HbA1c), triglycerides, total cholesterol, low density lipoprotein cholesterol (LDL-C), creatinine, urea and uric acid. Glucose measurements were obtained using the glucose oxidase method (intra-assay coefficient of variance [VC] $2.1 \%$, intra-assay CV 2.6\%). HbA1c was determined through high-pressure lipid chromatography. Total cholesterol, LDL-C, HDL-C and triglycerides levels were determined using direct enzymatic methods (Parsazmoon, Karaj, Iran). Samples were stored in the freezer at $-20^{\circ} \mathrm{C}$ before plasminogen activator inhibitor-1(PAI-1) measurement. PAI-1 activity was determined using enzyme-linked immune-sorbent assay (ELISA) technology. Creatinine was measured using the calibrated Jaffe method, (Parsazmoon, Karaj, Iran, intra assay CV=3.3\%). Urea was measured using a colorimetric assay (Parsazmoon, Karaj, Iran). Uric acid was measured through the calorimetric method (intra assay $\mathrm{CV}=1.27$ ), while measurements of urinary protein excretion were obtained through timed 24-hour urine collection. Urine protein was measured through immunoturbidimetry (intra-assay $\mathrm{CV}=2.1 \%$ ), and glomerular filtration rate (GFR) was calculated using the modification of diet in renal disease (MDRD) formula [12].

Statistical analysis: Continuous variables were presented as mean \pm standard error of Mean (SME). Categorical variables are presented as number and percentage. A chi-squared test and paired sample t-test were used to make group comparisons as indicated earlier. A conditional logistic regression model was utilized to evaluate outcomes (microalbuminuria and normoalbuminuric) in the studied population. Input variables were: PAI-1, HDL-C, duration of diabetes, systolic blood pressure, diastolic blood pressure, FBS, HbA1c, GFR, triglycerides, total cholesterol and LDL-C. Results with a p-value of $<0.05$ were considered significantly significant. Statistical Package for the Social Sciences (SPSS for windows, version 19, Chicago, IL), a software program, was used to complete the analysis. 


\section{RESULTS}

Primary characteristics of the study population are presented in Table 1 . The only observed differences between the cases and controls were in plasma uric acid and the usage of oral antihypertensive drugs. No significant differences were observed in matching variables between the two groups (Table 1).

Table 1. Baseline Characteristics of study population

\begin{tabular}{|c|c|c|c|}
\hline & Case $(n=38)$ & Control( $(\mathrm{n}=38)$ & P-value \\
\hline Gender (male, female) & $(22,16)$ & $(22,16)$ & - \\
\hline Age (years) & $58.47 \pm 1.69$ & $57.78 \pm 1.51$ & NS \\
\hline $\operatorname{BMI}\left(\mathrm{kg} / \mathrm{m}^{2}\right)$ & $27.15 \pm .74$ & $26.31 \pm .73$ & NS \\
\hline Waist circumference $(\mathrm{cm})$ & $95.88 \pm 2.91$ & $91.77 \pm 1.75$ & NS \\
\hline Duration of diabetes (years) & $10.69 \pm 1.53$ & $9.19 \pm 1.13$ & NS \\
\hline $\mathrm{SBP}(\mathrm{mmHg})$ & $122.42 \pm 3.02$ & $123.13 \pm 3.58$ & NS \\
\hline DBP (mmHg) & $71.54 \pm 1.63$ & $72.84 \pm 1.79$ & NS \\
\hline FBS (mg/dl) & $207.41 \pm 14.03$ & $196.58 \pm 15.08$ & NS \\
\hline HbA1C (\%) & $9.10 \pm 0.45$ & $8.62 \pm 4.24$ & NS \\
\hline $\mathrm{HbA} 1 \mathrm{C}(\mathrm{mmol} / \mathrm{mol})$ & $75.92 \pm 4.94$ & $70.67 \pm 4.24$ & NS \\
\hline Creatinine (mg/dl) & $1.15 \pm 0.05$ & $1.07 \pm 0.09$ & NS \\
\hline Urea $(g / 24 h)$ & $35.59 \pm 2.85$ & $33.36 \pm 2.99$ & NS \\
\hline Uric Acid (mg/dl) & $5.45 \pm .40$ & $3.80 \pm .45$ & $<0.05$ \\
\hline Total Cholesterol (mg/dl) & $187.07 \pm 8.53$ & $184.13 \pm 8.85$ & NS \\
\hline LDL-C (mg/dl) & $107.36 \pm 7.04$ & $105.74 \pm 6.79$ & NS \\
\hline HDL-C (mg/dl) & $39.31 \pm 1.48$ & $41.95 \pm 1.80$ & NS \\
\hline TG (mg/dl) & $190.90 \pm 22.15$ & $163.42 \pm 17.89$ & NS \\
\hline GFR (ml/min) & $65.61 \pm 3.04$ & $77.67 \pm 6.37$ & NS \\
\hline Microalbuminuria (mg/24h) & $192.44 \pm 20.97$ & $7.49 \pm 1.12$ & $<0.0001$ \\
\hline PAI-1 (ng/ml/h) & $35.97 \pm 2.46$ & $28.07 \pm 2.60$ & 0.02 \\
\hline IHD n $(\%)$ & $4(11)$ & $2(6)$ & NS \\
\hline \multicolumn{4}{|l|}{ Medication } \\
\hline Oral Hypoglycemic drug n (\%) & $28(74)$ & $33(87)$ & NS \\
\hline Insulin $\mathrm{n}(\%)$ & $12(31)$ & $8(21)$ & NS \\
\hline Antihypertensive $\mathrm{n}(\%)$ & $23(61)$ & $11(29)$ & $<0.01$ \\
\hline $\mathrm{ARB}(\mathrm{n})$ & 14 & 7 & \\
\hline ACEI (n) & 8 & 5 & \\
\hline CCB n $(\%)$ & 1 & 2 & \\
\hline beta-blocker $\mathrm{n}(\%)$ & 4 & & \\
\hline Lipid-lowering $n$ & $25(66)$ & $23(61)$ & NS \\
\hline$(\%)$ statin $(\mathrm{n})$ & 23 & 19 & \\
\hline fibrates $(\mathrm{n})$ & 3 & 4 & \\
\hline
\end{tabular}


Higher PAI-1 activity was associated with many factors, including high BMI and waist circumference, high triglyceride concentration, and high total HDL-cholesterol ratio. In addition, young age and a shortened duration of diabetes, two confounding factors, were also associated with higher PAI-1 activity. Through a multivariate linear regression analysis that adjusted for confounding factors, the strongest predictors of high PAI-1 activity were calculated. The levels of PAI-1 activity, waist circumference and BMI in the study groups are presented in Table 2. PAI-1 activity was significantly higher in the case group when compared to that of the control group $(p=0.02)$. There was no significant difference in BMI or waist circumference between the two groups. When comparing the case and control males, there was no significant difference found in PAI-1 activity between microalbuminuric and normoalbuminuric males. On the contrary, female participants with microalbuminuria had higher PAI-1 activity levels when compared to the normoalbuminuric controls, while there was no significant difference in BMI and waist circumference between the two groups (table 2).

Table 2. PAI-1 levels, Waist circumference, BMI and CVD in study groups according to gender

\begin{tabular}{|c|c|c|c|c|}
\hline & & Case $(n=38)$ & Control $(n=38)$ & p-value \\
\hline \multirow[t]{3}{*}{ PAI-1 (ng/ml) } & Male & $29.04 \pm 2.69$ & $26.04 \pm 2.22$ & 0.35 \\
\hline & Female & $45.06 \pm 3.35$ & $20.75 \pm 5.29$ & 0.04 \\
\hline & Total & $35.95 \pm 2.46$ & $28.07 \pm 2.60$ & 0.02 \\
\hline \multirow[t]{3}{*}{ W.C (cm) } & Male & $92.80 \pm 9.56$ & $91.40 \pm 10.02$ & 0.648 \\
\hline & Female & $99.13 \pm 24.55$ & $92.56 \pm 10.87$ & 0.338 \\
\hline & Total & $95.88 \pm 2.91$ & $91.77 \pm 1.75$ & 0.567 \\
\hline \multirow[t]{3}{*}{ BMI $\left(\mathbf{k g} / \mathbf{m}^{2}\right)$} & Male & $25.56 \pm 3.59$ & $24.66 \pm 3.24$ & 0.396 \\
\hline & Female & $29.12 \pm 4.93$ & $28.84 \pm 4.93$ & 0.875 \\
\hline & Total & $27.15 \pm 0.74$ & $26.31 \pm 0.73$ & 0.567 \\
\hline \multirow[t]{3}{*}{ CVD (\%) } & Male & $2(9.9)$ & $2(9.9)$ & 0.80 \\
\hline & Female & $3(18.7)$ & $0(0 \%)$ & 0.06 \\
\hline & Total & $6(15.7)$ & $5(13.1)$ & 0.80 \\
\hline
\end{tabular}

Table 3 shows the results of a conditional logistic regression model which considered albuminuria as the outcome variable. There was a positive correlation between PAI-1 activity and microalbuminuria (OR: 1.462, p-value: 0.041). 
Table 3. Conditional logistic regression was employed to study variables influencing Microalbuminuria in patients with T2D

\begin{tabular}{|l|l|l|c|}
\hline & Odds Ratio & $95 \%$ Confidence interval & P-value \\
\hline PAI-1 & 1.462 & $1.017-2.102$ & .041 \\
\hline HDL-C & .988 & $.892-1.094$ & .816 \\
\hline Duration of diabetes & 1.049 & $.936-1.175$ & .409 \\
\hline BMI & 1.339 & $.933-1.922$ & .114 \\
\hline Age & 1.127 & $.881-1.440$ & .341 \\
\hline SBP & 1.005 & $.962-1.050$ & .818 \\
\hline
\end{tabular}

Figures 1 and 2 show the PAI-1 activities of diabetic men and women respectively, while also comparing the cases (microalbuminuric) and controls (normoalbuminuric).

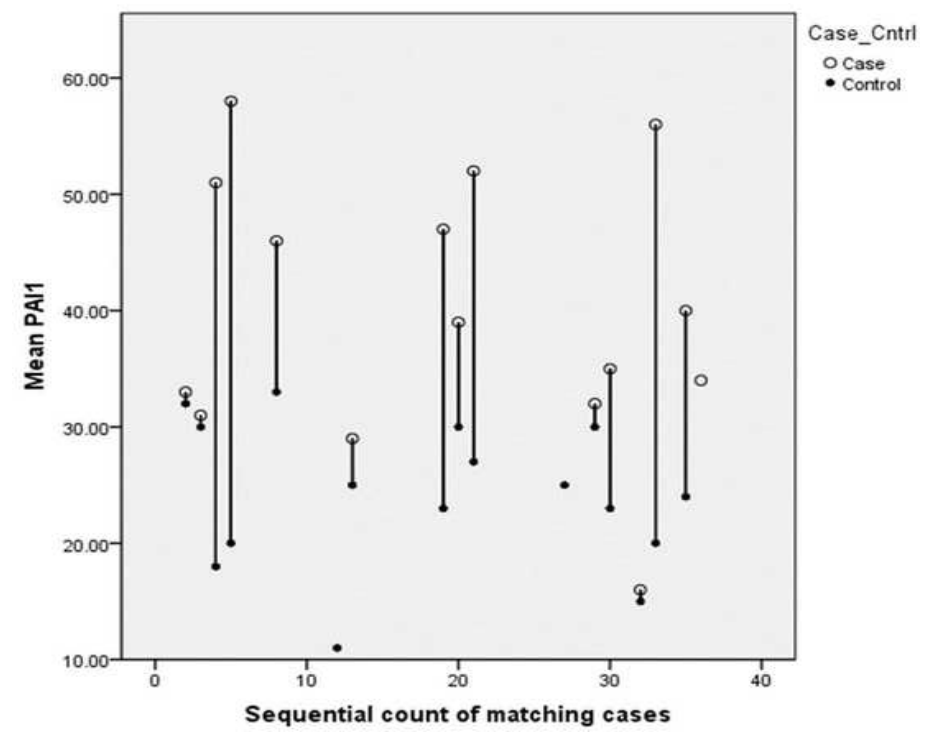

Figure 1. Presenting plasma PAI-1 activities in pairs of diabetic women with albuminuria (cases) and without albuminuria (controls)

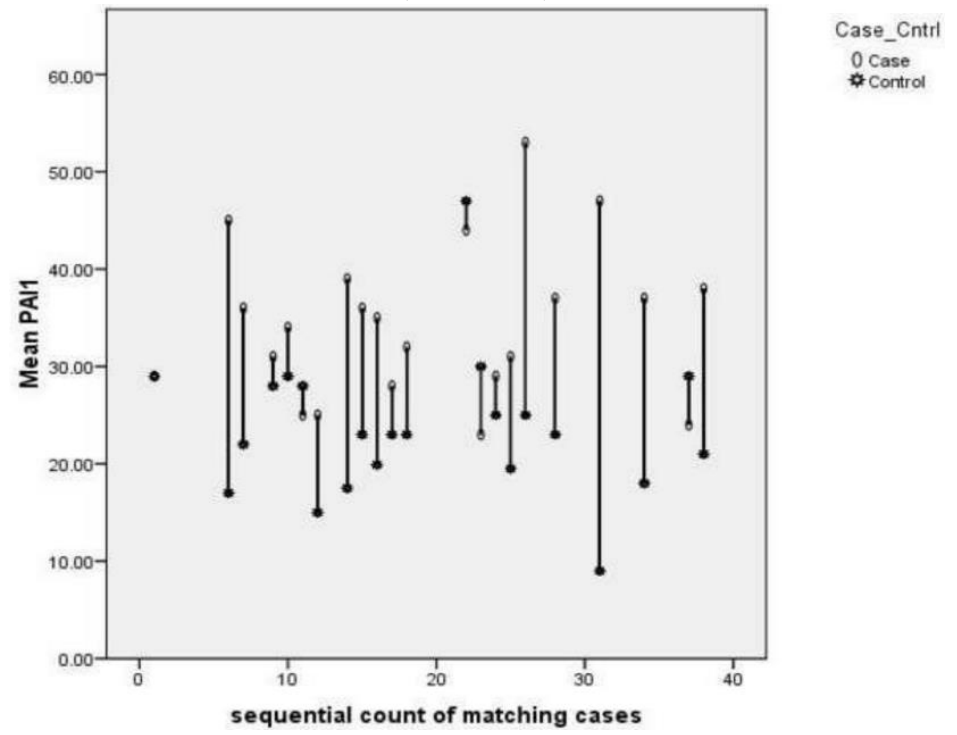

Figure 2. Presenting plasma PAI-1 activities in pairs of diabetic men with albuminuria (cases) and without albuminuria (controls). 


\section{DISCUSSION}

The main finding of this study was that diabetic women with microalbuminuria have significantly increased PAI-1 activity in comparison to diabetic women with normoalbuminuria. This result was not observed in the male participants. Many studies have found that PAI-1 activity increases in patients with T2D [10,14]. Increased PAI-1 activity is an important predictor for developing microangiopathy and endothelial damages [15]. Moreover, dysfunction of fibrinolysis has been suggested as a possible link between microalbuminuria and cardiovascular disease [16].

Animal studies demonstrate that PAI-1 contributes to diabetic nephropathy by regulating TGF- $\beta$ and renal extra matrix production. They suggested that PAI-1 suppression through fenofibrate or angiotensin converting enzyme inhibitors (ACEI) delayed the progression of diabetic glomerular injury in experimental diabetes $[17,18]$. Susanne et al. (2005) reported PAI-1 deficiency retarded diabetic nephropathy in rats. They believed that suppression of PAI-1 activity may be a therapeutic target in diabetic nephropathy [9].

On the other hand, abnormalities of hemostasis and the fibrinolysis system are strongly associated with atherosclerotic disease [19]. Kario et al. reported PAI -1 activity increased in diabetic patients with microalbuminuria compared to those with normoalbuminuria, suggesting endothelial cell damage. [20].

The effect of gender on the relationship between PAI-1 activity and microalbuminuria has not been previously determined. It is possible that fibrinolysis impairment, due to elevated PAI-1 activity in the early stages of diabetic nephropathy, may be more prominent in women than men.

Women have significantly higher plasma levels of total cholesterol, TG, LDL-C, HDL-C, lipoprotein A and apolipoprotein B [21, 22]. Furthermore, urinary albumin excretion, which is more severely pronounced in women, is associated with insulin resistance and cardiovascular risk factors in patients with type 2 diabetes [23]. Insulin resistance is also significantly correlated with elevated PAI-1 level, waist circumference, BMI, triglyceride levels, fasting blood glucose and HbA1c in type 2 diabetes [16, 24].

In addition, increasing evidence shows that obesity increases PAI-1 production in ectopic fat tissue, while weight loss decreases the production [25, 26]. Elevated PAI-1 activity in women with android fat distribution could be mediated by sex hormones, as postmenopausal hormone replacement results in decreased PAI-1 activity [27, 28]. Saad et al. (2012) found that women with type 2 diabetes have a pro-thrombotic fibrin profile which exhibits gender-specific associations with anthropometric and metabolic factors [11]. Furthermore, Stefan et al. demonstrated that denser fibrin clots and resistance to fibrinolysis in women, which is not fully explained by fibrinogen or PAI-1 activity, may contribute to the loss of cardiovascular protection in females with diabetes [23]. Moreover, increased waist circumference, BMI and lower HDL-C levels in diabetic women with albuminuria were associated with a pro-thrombotic clot phenotype [11,29]. The increased amount of visceral fat in women with type 2 diabetes may contribute to increased PAI-1 activity and subsequent increased risk for thrombo-vascular disease, regardless of BMI and total fatness [30]. However, it has been suggested that visceral adiposity could lead to portal nonstratified fatty acids (NEFA) accumulation, which may stimulate hepatic PAI-1 production. The inhibition of lipolysis is mainly linked to plasma insulin concentrations but may be impaired in those with diabetes [27]. 
On the other hand, PAI-1 is classified as an acute phase protein, and inflammatory cytokines have been shown to induce PAI-1 production in endothelial cells [25] and in the liver [31]. The same cytokines have been shown to promote vascular inflammation and atherosclerosis [25]. Women have been shown to secrete higher levels of inflammatory cytokines, such as interleukin 6, and have a greater response to stress [32]. Moreover, hyperglycemia-induced oxidative stress and reduced levels of $\mathrm{NO}$ within the vasculature have been suggested to increase PAI-1 production [33]. Previous studies have shown that patients with albuminuria have higher serum heat shock protein 70 (HSP-70) levels. Levels of these heat shock proteins, which are markers of oxidative stress, are significantly higher in diabetic women in comparison with diabetic men [32]. In addition, diabetes induces impaired cellular defense mechanisms against oxidative stress, which is more prominent in women [33]. Therefore, diabetic women are more susceptible to endothelial damages, which act as predisposing risk factors for complications of type 2 diabetes.

Conclusively, in women with type 2 diabetes and albuminuria, PAI-1 activity was independently and significantly associated with urinary albumin excretion. Elevated PAI-1 activity is partly responsible for susceptibility to diabetic complications in women when compared with men. Different effects of sex hormones in inflammatory conditions could be a possible explanation.

Although this study was a well-designed, matched case-control study, its main limitation was an inadequate sample size for predicting exact correlations. In addition, determining whether increased PAI-1 activity leads to microalbuminuria, or vice versa, remains unknown. The authors suggest further cohort studies to determine whether such relationship exists.

List of Abbreviations: BMI, body mass index; SBP, Systolic blood pressure; DBP, diastolic blood pressure; FBS, fasting blood sugar; HbA1C, hemoglobin A1C; LDL-C, low density lipoprotein cholesterol; HDL-C, high density lipoprotein cholesterol; TG, Triglyceride; GFR, glomerular filtration rate; PAI-1, plasminogen activator inhibitor 1; IHD, ischemic heart disease; ACEI, angiotensin converting enzyme inhibitor; $\mathrm{ARB}$, aldosterone receptor blocker; $\mathrm{CCB}$, calcium channel blocker.

Competing interests: The authors declare that there are no conflicts of interest.

Authors' contributions: M.I, M.N, H.M and A.E participated in the design of the study, data collection, statistical analysis, and drafting of the manuscript. P.H, Sh.P, S.R participated in supervision and provided oversight when the manuscript was being drafted. R.H, P.H provided advice regarding development of the protocol for the study and assisted in the design of the study.

Acknowledgment: There are no acknowledgements.

\section{REFERENCES:}

1. Wiman B, Hamsten A: the fibrinolytic enzyme system and its role in the etiology of thromboembolic disease. Semin Thromb Hemost 1990, 16(3):207-216.

2. Zandbergen AA, Sijbrands EJ, Lamberts SW, Bootsma AH: Normotensive Women With Type 2 Diabetes and Microalbuminuria Are at High Risk for Macrovascular Disease. Diabetes Care 2006, 29(8):1851-1855. 
3. Asplund-Carlson A, Hamsten A, Wiman B, Carlson LA: Relationship between plasma plasminogen activator inhibitor-1 activity and VLDL triglyceride concentration, insulin levels and insulin sensitivity: studies in randomly selected normo- and hypertriglyceridaemic men. Diabetologia 1993, 36(9):817-825.

4. Schneider DJ, Nordt TK, Sobel BE: Attenuated fibrinolysis and accelerated atherogenesis in type II diabetic patients. Diabetes 1993, 42(1):1-7.

5. Eriksson M, Zethelius B, Eeg-Olofsson K, Nilsson PM, Gudbjornsdottir S, Cederholm J, Eliasson B: Blood lipids in 75,048 type 2 diabetic patients: a population-based survey from the Swedish National diabetes register. Eur J Cardiovasc Prev Rehabil 2010, 18(1):97-105.

6. Landin K, Stigendal L, Eriksson E, Krotkiewski M, Risberg B, Tengborn L, Smith U: Abdominal obesity is associated with an impaired fibrinolytic activity and elevated plasminogen activator inhibitor-1. Metabolism 1990, 39(10):1044-48.

7. Galajda P, Kubisz P, Mokan M: A multicomparmental and multifactorial model of production of plasminogen activator inhibitor-1 (PAI-1). Experimental studies 1998, 44(12):718-721.

8. Mehta R, Shapiro AD: Plasminogen activator inhibitor-1 deficiency. Haemophilia 2008, 14(6):1255-60.

9. Nicholas SB, Aguiniga E, Ren Y, Kim J, Wong J, Govindarajan N, Noda M, et al.: Plasminogen activator inhibitor-1 deficiency retards diabetic nephropathy. Kidney Int 2005, 67(4):1297-1307.

10. Maser RE, Ellis D, Erbey JR, Orchard TJ: Do tissue plasminogen activator inhibitor-1 complexes relate to the complications of insulin-dependent diabetes mellitus? Pittsburgh epidemiology of diabetes study. J Diabetes Complications 1997, 11(4):243-249.

11. Alzahrani SH, Hess K, Price JF, Strachan M, Baxter PD, Cubbon R, Phoenix F, et al.: Gender specific alteration in fibrin structure function in type 2 diabetes: Associations with cardiometabolic and vascular markers. J Clin Endocrinol Metabol 2012, 97(12):2282-2287.

12. Berrut G, Bouhanick B, Fabbri P, Guilloteau G, Bled F, Le Jeune JJ, Fressinaud P, et al.: Microalbuminuria as a predictor of a drop in glomerular filtration rate in subject with noninsulin dependent diabetes mellitus and hypertension. Clin Nephrol 1997, 48(2):92-97.

13. Keane WF, Brenner BM, de Zeeuw D, Grunfeld JP, McGill J, Mitch WE, Ribeiro AB, et al.: The risk of developing end-stage renal disease in patients with type 2 diabetes and nephropathy. Kidney Int 2003, 63(4):1499-1507.

14. Chudy P, Kotulichova D, Stashko J, Kubisz P: The relationship among TAF1, T-PA, PAI1 and $\mathrm{F} 1+2$ in type 2 diabetic patients with normoalbuminuria and microalbuminuria. Blood Coagul Fibrinolysis 2011, 22(6):493-498.

15. Eddy AA: Plasminogen activator inhibitor-1 and the kidney. Renal Physiol 2002, 283(2):203-220.

16. Ahmed S, Ahmad SA: Gender Difference and Relationship of Insulin Resistance with Microalbuminuria Type-2 Diabetes. J Coll Physicians Surgeons Pakistan 2010, 20(1):2632.

17. Chen LL, Zhang JY, Wang BP: Renoprotective effects of fenofibrate in diabetic rats are achieved by suppressing kidney PAI-1. Vascul Pharmacol 2006, 44(5):309-315

18. Kenichi M, Masanobu M, Takehiko K, Shoko T, Akira F, Katsushige A, Takashi H, et al.: Renal synthesis of urokinase type plasminogen activator, its receptors, and PAI-1 in diabetic 
nephropathy in rats: modulation by angiotensin converting enzyme inhibitor. J Lab Clin Med 2004, 144(2):69-77.

19. Agewall S, Lindstedt G, Fagerberg B: Independent relationship between microalbuminuria and plasminogen activator inhibitor-1 activity (PAI-1) activity in clinically healthy 58- yearold men. Atherosclerosis 2001, 157(1):197-202.

20. Kario K, Matsuo T, Kobayashi H, Matsuo M, Sakata T, Miyata T: Activation of tissue factor induced coagulation and endothelial cell dysfunction in non-insulin-dependent diabetic patients with microalbuminuria. Arterioscler Thromb Vasc Biol 1995, 15(8):1114-20.

21. Carnevale Schianca GP, Fra GP, Colli E, Bigliocca M, Mella R, Scaglia E, Bartoli E: Sex differences in lipid profiles in relation to the progression of glucose abnormalities. J Diabetes 2012, 4(1):95-101.

22. Eriksson M, Zethelius B, Eeg-Olofsson K, Nilsson PM, Gudbjornsdottir S, Cederholm J, Eliasson B: Blood lipid in 75048 type 2 diabetic patients: a population based survey from the Swedish National diabetes register. Eur J Cardiovasc Prev Rehabil 2010, 18(1):97-105.

23. Brandenburg SL, Lindenfeld J, Reusch JE, Regensteiner JG: Cardiovascular risk in women with type 2 diabetes. Med Clin North Amer 2003, 87:955-969.

24. Galajda P, Sutarik L, Vladar L, Stasko J, Kubisz P, Mokan M: Plasminogen activator inhibitor (PAI-1) and diabetes mellitus. I. Regulation of PAI-1 levels. Vintr Lek 1997, 44(3):804-8.

25. Vaughan DE: PAI-1 and atherothrombosis. J Thromb Haemost 2005, 3(8):1879-83.

26. Juhan-Vague I, Alessi MC, Mavri A, Morange PE: Plasminogen activator inhibitor-1, inflammation, obesity, insulin resistance and vascular risk. J Thromb Haemost 2003, 1(7):1575-9.

27. Toft I, Bonaa KH, Ingebretsen OC, Nordoy A, Birkeland KI, Jenssen T: Gender Differences in the Relationships Between Plasma Plasminogen Activator Inhibitor-1 Activity and Factors Linked to the Insulin Resistance Syndrome in Essential Hypertension. Arterioscler Thromb Vasc Biol 1997, 17(3):553-9.

28. Hauner H, Ditschuneit HH, Pal SB, Moncayo R, Pfeiffer EF: Fat distribution, endocrine and metabolic profile in obese women with and without hirsutism. Metabolism 1988, 37(3):2816.

29. Nakhjavani M, Morteza A, Jenab Y, Ghaneei A, Esteghamati A, Karimi M, Farokhian A: Gender difference in albuminuria and ischemic heart disease in type 2 diabetes. Clin Med Res 2012, 10(2):51-6.

30. Mertens I, Van der Planken M, Corthouts B, Wauters M, Peiffer F, De Leeuw I, Van Gaal L: Viseral fat is a determinant of PAI-1 activity in diabetic and nondiabetic overweight and obese women. Horm Metab Res 2001, 33(10):602-7.

31. Meshkani R, Adeli K: Hepatic insulin resistance, metabolic syndrome and cardiovascular disease. Clin Biochem 2009, 42(13-14):1331-46.

32. Nakhjavani M, Morteza A, Meysamie A, Esteghamati A, Khalilzadeh O, Esfahanian F, Khajeali L, et al.: Serum heat shock protein 70 and oxidized LDL in patients with type 2 diabetes: does sex matter? Cell Stress Chaperones 2010, 16(2):195-201.

33. White RE, Gerrity R, Barman SA, Han G: Estrogen and oxidative stress: A novel mechanism that may increase the risk for cardiovascular disease in women. Steroids 2010, 75(11):788-793. 